Acta Hispanica (2020) Supplementum II: 655-662

\title{
AMEREIDA: REALIDAD CULTURAL ABIERTA PARA ENCUENTROS Y COLABORACIÓN ${ }^{1}$
}

\author{
MAgDALENA BARBARUK
}

Universidad de Wroclaw

Resumen: El objetivo del artículo es la presentación de Amereida desde la perspectiva de los estudios culturales. Según la autora, es un fenómeno que puede servir como modelo de ambiguas relaciones de América Latina y Europa, estrechamente vinculadas a la idea de la comunidad y la cooperación. Las ideas de Amereida se tradujeron en la llamada "travesía" (un viaje del grupo de arquitectos, poetas, artistas de América y Europa que atravesaron el continente americano en el año 1965 con el propósito de la descolonización cultural) y se realizan desde el año 1970 en la "Ciudad Abierta" (Chile). Amereida, aunque parece dependiente de los patrones occidentales (el neologismo "Amereida" es una combinación de "América" y "Eneida"), es más bien un ejemplo del latinoamericanismo moderno. En conclusión, Barbaruk postula el uso de otras herramientas teóricas de índole heterogénea para describir el fenómeno de Amereida. Se trata de ideas tomadas de A. Rimbaud ("yo es otro"), G. Deleuze, F. Guattari (literatura menor), M. Bajtín (exotopía), J. Derrida (espectro) o el concepto de la sutura.

Palabras clave: Amereida, Europa, Latinoamérica, travesía.

Abstract: The objective of the article is Amereida's presentation from the perspective of cultural studies. According to the author, it is a phenomenon that can serve as a model of ambiguous relations in Latin America and Europe, which are closely related to the idea of community and cooperation. Amereida's idea was established in "crossing" (a trip by the group of architects, poets, artists from America and Europe that crossed the American continent in 1965 for its cultural decolonization) and have been carried out since 1970 in "the Open City" (Chile). Amereida although it seems dependent on Western patterns (neologism "Amereida" is a combination of "America" and "Aeneid") is rather an example of modern Latin Americanism. In conclusion Barbaruk postulates the use of other, heterogeneous theoretical tools to describe the Amereida phenomenon: ideas taken from A. Rimbaud ("I is another"), G. Deleuze, F. Guattari (minor literature), M. Bajtín (exotopia), J. Derrida (spectre) or suture concept.

Keywords: Amereida, Europe, Latin America, Crossing.

\footnotetext{
${ }^{1} \mathrm{El}$ texto fue preparado como parte de la beca de investigación recibida del Centro Nacional de Ciencias "Trayectorias de palabras. Impacto cultural de Amereida” (No. 2017/25/B/HS2/00453).
} 
Amereida: realidad cultural abierta para encuentros y colaboración

\section{Tierras de los encuentros y la necesidad de la literatura}

Nací en Wrocław, una ciudad del sur de Polonia, llamada "ciudad de los encuentros". La metáfora, utilizada por el papa Juan Pablo II en 1997 y retomada por los gobiernos locales, mostraba cómo la trágica historia de la ciudad tras la Segunda Guerra Mundial con el cambio de las fronteras del Estado y un cambio casi total de la población-, terminó transformándose en la virtud de la multiculturalidad y la apertura. La identidad actual de Wrocław ha sido formada por personas que, a consecuencia del cambio de las fronteras, fueron expatriadas por fuerza de sus casas en tierras polacas que hoy en día pertenecen a Ucrania, y personas procedentes de otras partes de Polonia. Mi ciudad, que durante la guerra fue transformada en una fortaleza, la Festung Breslau, quedó destruida en un $70 \%$ y hubo que reconstruirla en todos los planos de la vida comunitaria: el cultural, el social y el de la civilización. Ese esfuerzo se llevó a cabo de la mano de personas que habían llegado a una "ciudad ajena" cuya pertenencia a la nueva Polonia fue puesta en duda durante largo tiempo.

El susodicho contexto es importante para entender por qué me dediqué a estudiar el continente llamado Nuevo Mundo, el cual, en la época moderna, ha sido formado por los llegados desde fuera y el cual ha surgido desde diversos gestos de constitución radicales (de manera que el "mundo" que sirve de punto de referencia para mi reflexión sobre Latinoamérica es una ciudad de la Europa central). En Wrocław, pese al sentimiento de la precariedad y a la falta de la soberanía política, se consiguió crear un modo de vida peculiar; por ello me fascinó Amereida, un proyecto utópico que se propuso volver a fundar América "habitándola poéticamente". En ambos lugares, ante un vacío cultural, se declaró la fe en la palabra literaria. El filólogo Tadeusz Mikulski, llegado a Wrocław en 1945, dijo: "hacen falta cien plumas para expresar el retorno de lo polaco a la ciudad" (Mikulski, 1961: 28). Sus palabras sonaban como un eco de la propaganda comunista de las "Tierras Recuperadas", en cuya capital se convirtió Wrocław (que había sido ciudad polaca solo hasta mediados del siglo XIV); pero demostraban fe en la fuerza impulsora y generadora de la literatura.

La literariedad de Wrocław y de la región de Baja Silesia se pone en tela de juicio, pues han pasado a ser centros de la literatura fantástica; castillos de la época alemana se volvieron castillos de Hogwarth y pintorescos valles, lugares de puesta en escena de aventuras de las novelas de Tolkien. En Baja Silesia también se encuentra el pueblo de don Quijote, Wojcieszyn. Sus habitantes construyeron molinos de viento, quijotizan visualmente el espacio y realizaron una adaptación cinematográfica de la novela de Cervantes, a quien encarnó, por cierto, la alcaldesa del pueblo. Estos fenómenos causan indignación de los tradicionalistas pero dan pie a afirmar un universalismo peculiar de Baja Silesia. Se trata de un palimpsesto de unas capas textuales inesperadas. Comparada con él, la identidad cultural de América del Sur aparece, paradójicamente, como más auténtica, al haber sido determinada por la historia y, en particular, por el período colonial. No por ello deja de suscitar dudas y controversias. 
Magdalena Barbaruk

\section{Amereida: ¿La herencia europea o europocéntrica?}

La primera de las controversias está relacionada con el propio neologismo de "Amereida", el cual designa un proyecto de la descolonización cultural de América creado en Chile, de la mano del poeta Godofredo Iommi Marini y el arquitecto Alberto Cruz Covarrubias. La palabra viene de la unión de "América" y "Eneida", y supone que el contexto para la nueva y deseada tradición sea la epopeya de Virgilio. No eran los únicos componentes "europeos" de "Amereida", pues la historia del grupo es la de diversos encuentros y trabajos compartidos. La comunidad con la que se identificaba Amereida no era nacional ni étnica. Al caracterizar Amereida, no es difícil caer en la simplificación y el malentendido; Iommi (o "Godo") cita un ejemplo de ello, al contar una historia de su estancia en Múnich, en 1963. Al concluir una de sus conferencias, se le acercó una joven y preguntó:

usted, que es un americano, por qué nos viene a decir a nosotros los europeos qué es
lo que tenemos que hacer (porque yo hablaba de Hölderlin y de Novalis
[...]). Entonces, la niña largándose me dijo: y ¿qué es lo que son
ustedes? ¿quienes son ustedes? ¿ustedes son una especie como de
secreción de inmigrantes europeos? Estas eran preguntas para
descalificarme, es decir, cómo te permites venir a decirme quién soy yo cuando
tú eres apenas un recién nacido que no tiene origen. Yo me quedé perplejo. Es
cierto. Entonces, me acordé de Virgilio, el poeta que escribió la Eneida
y diciendo... [...] estas musas que están en Grecia, yo las voy a llevar a Roma
y lo que él escribió - la Eneida y todo eso- es una apología de Roma.
¿Qué es lo que nosotros somos en virtud de la herencia europea? ¿qué
es lo que hemos asimilado y qué es lo propio y característico de
nosotros? De ahí nació la hospitalidad, de ahí nació todo eso. Ahí
empezó a esbozarse Amereida (Iommi, 1999).

Amereida se fue creando con referencia a Europa, pero ¿significa esto que fue europocéntrica? Iommi, argentino de origen italiano, se sentía heredero de la gente latina. En su Eneida-Amereida (1982) escribió que Eneas "es un emigrante". Para Iommi, el exilio y la consiguiente necesidad de desplazar la patria a Roma fueron el fundamento de la cultura latina. Dice así: "La latinidad surge pues de este transporte, en palabra griega, de una metáfora que surge del lenguaje griego y se posa en el latín” (Iommi, 1982: 11). Roma era para él símbolo de la apertura y de la hospitalidad, y crisol de la otredad. De su infancia guardaba el recuerdo de un gran mapa de Grecia en el muro de su colegio y el de saberse de memoria la Divina comedia de Dante. Solo después de abandonar sus estudios universitarios y marcharse de Buenos Aires a Brasil, empezó a conocer la poesía latinoamericana, la poesía "desmelenada", lo cual supuso para él una experiencia de shock y un punto de inflexión. 
Amereida: realidad cultural abierta para encuentros y colaboración

La creación de Amereida se desprende de la idea de que, en medio del continente, existe un "mar interno" desconocido que se debe habitar "poéticamente", es decir, de manera permanente, fáctica y esencial. La experiencia de la falta, una añoranza extraña e indeleble, influye en que el espacio en el que vivimos se vuelva fluido, móvil, desplazado, heterogéneo y heterotópico. Según el grupo de Valparaíso, la falta debía encontrar su respuesta en la poesía. La frase clave para Amereida, "poéticamente habita el hombre en esta tierra", proviene de la poesía de Friedrich Hölderlin ("dichterisch wohnet der Mensch"), sometida a la famosa interpretación de Martin Heidegger. Igualmente, la concepción de la apertura del espacio a través del acto poético, la llamada phalène, bebe de la misma fuente; destaquemos el hecho de que se trate de una fuente alemana. La idea de liberar la poesía de la escritura, así como el propósito de inscribirla en una acción colectiva llevada a cabo en el espacio público, se fue desarrollando durante mucho tiempo, en Buenos Aires, en Brasil, en Santiago de Chile pero las pruebas decisivas las realizó Iommi en París, en los años 50 del siglo pasado. La idea presenta influencias del surrealismo francés, de Arthur Rimbaud, quien percibía la poesía como una herramienta para cambiar la vida, y también influencias del pensamiento pedagógico alternativo de Brasil, con la importancia que en él se concede al juego.

La manifestación más radical de la concepción de las phalènes, de su capacidad impulsora ontológica y axiológicamente, fue la llamada travesía, un viaje efectuado en 1965 desde la Tierra del Fuego hasta Santa Cruz de la Sierra, en Bolivia; esta ciudad fue proclamada la nueva capital poética del continente y su elección fue consecuencia de la yuxtaposición de las líneas de la Cruz del Sur sobre el mapa invertido de América. Aunque la travesía tenía un carácter descolonizador, también participaron en ella europeos: Michel Deguy, François Fédier, Henri Tronquoy y Jonathan Boulting. En su transcurso, fue surgiendo el poema Amereida, obra colectiva, en la cual, además de recurrir a la tradición de la cultura latina, se remitía a las crónicas coloniales españolas.

Cinco años después del regreso de la travesía, Cruz, Iommi y sus colaboradores de la Escuela de Arquitectura y Diseño de la Universidad Católica de Valparaíso, creada por ellos en 1952, fundaron en las dunas de Ritoque la Ciudad Abierta, un lugar donde poder realizar la idea de la unidad del trabajo y la vida. En las bases de dicha idea está la tradición de la colonia artística, en su variante del siglo XIX y en la modernista (Bauhaus), y la de las misiones jesuíticas; también, la concepción de la ciudad como espacio sagrado (en la Ciudad Abierta existe un cementerio en el que yacen los fundadores de Amereida junto a sus familias) y la utopía entendida como un lugar feliz (eutopía). La visión de la Escuela de Valparaíso era distinta de la estética del modernismo europeo. No le interesaba una imitación externa de formas arquitectónicas, a manera de Sergio Larraín, por ejemplo, autor del edificio Oberpaur de Santiago (1929), inspirado en los Almacenes Schocken de Chemnitz, diseñados por Erich Mendelsohn (1928). La arquitectura debía fortalecer la conciencia de ser americanos y brotar de la vida, estrechamente unida a la poesía y el arte. El grupo no compartía la fe optimista en el progreso tecnológico, usaba materiales reciclados. Al destacar la primacía del proceso de la realización de un proyecto sobre su 
resultado, denunciaba los fetiches de la productividad, de la obra y del autor. Sus construcciones, las llamadas hospederías, no llevaban firma alguna, por considerarse obra colectiva. En su visión, la ciudad debía ser "abierta", hospitalaria e inestable, por depender de las interpretaciones cambiantes de la vida. Dijo Iommi:

\begin{abstract}
Me acuerdo cuando Alberto inventó la arquitectura coral, es decir, no hecha por uno, sino por todos. Esa es la cosa de Lautréamont en los poemas, entonces cada uno aportaba lo suyo. Eso viene directamente de la poesía. Yo inventé la Phálene por eso, porque dije ¿cómo puedo hacer la poesía con otros? así como Sócrates, le haces deducir un poema, como un partero, le sacas del fondo de las cosas todas sus posibilidades (1999).
\end{abstract}

Amereida es moderna pero no lo es a la manera "cartesiana". ¿Puede afirmarse que su carácter corrobora la tesis de lo barroco de la modernidad latinoamericana, predicada por José Lezama Lima, Alejo Carpentier y Octavio Paz, entre otros? Esta cualidad se manifiesta a través del mestizaje, del sincretismo cultural, a través de ritos de sacrificio que, en su variante secularizada, cobran formas teatrales o lúdicas, así como a través de la tendencia a la utopía (Nawrocka, 2010: 178). Todos estos elementos se dan en Amereida, pero la fórmula presenta ciertos límites; a pesar de todo, conlleva valoración y es demasiado lejana a la axiología de la modernidad: a la apertura, al deseo del cambio de la vida y a la autonomía del arte.

\title{
3. Amereida y el latinoamericanismo moderno
}

Amereida también puede entenderse como un fenómeno que representa el latinoamericanismo moderno, surgido a principios del siglo XX, así como su versión elitista, el llamado arielismo, cuyo nombre honra el famoso ensayo Ariel de José Enrique Rodó. En este caso, el argumento clave sería el hecho de vincular Amereida a la Antigüedad mediterránea. Al comienzo del siglo XIX, la unidad del continente suramericano se pensaba desde la perspectiva del antagonismo entre Latinoamérica y Europa; a su vez, un siglo más tarde, resultó ser más importante destacar la particularidad del continente frente a Estados Unidos. A principios del siglo XX, en Latinoamérica se empezó a criticar el pragmatismo, el utilitarismo relacionado con la industrialización y la urbanización, y se dio un cambio de rumbo hacia los valores espirituales. Los dolores de la modernidad se identificaron con los Estados Unidos; y la tradición espiritual-moral, con la cultura mediterránea. Según los partidarios del arielismo, Latinoamérica, a través de España, se convirtió en heredera de Grecia y Roma, de manera que debía reconocer el fundamento de su identidad cultural como latino, universalista y utópico. América, en su autoidentificación, pasó a formar una parte de la cultura universal, que debía determinarse dentro de la cultura occidental. El latinoamericanismo moderno se basa en la idea del cosmopolitismo grecocristiano, es decir, en la visión de una unidad global 
Amereida: realidad cultural abierta para encuentros y colaboración

conformada por diversos contenidos culturales nacidos como referencia local a mitos universales. Existe más de una razón para discrepar de esa visión latinoamericana de Amereida. El quid de la modernidad promovida por Amereida es un estilo de vida enmarcado en un espacio concreto y en una comunidad. Lo descrito en el diario de la travesía de 1965, actos poéticos, situaciones inesperadas, encuentros con personas reales, dotaba de sentidos nuevos lugares conocidos solo por autóctonos o lugares deshabitados. Esto permite ver el territorio americano desde una perspectiva más amplia; a la vez, dado el carácter abierto y situacional de la práctica creadora del grupo, esta última no puede reducirse a una interpretación local de motivos culturales universales.

\section{Perspectivas de reflexión}

El objetivo de la acción de "mapear" la heterogeneidad cultural de Amereida no es denunciar lo colonial de sus prácticas o demostrar que deconstruían contenidos europeos. Un gesto de protesta no siempre supone oposición y lucha basada en el orden binario: nosotros-vosotros. También puede ser una manera de minar dicha oposición, a través de su transgresión o de su lectura subversiva. Puede efectuarse en el espíritu de la "literatura menor" de Gilles Deleuze y Félix Guattari, donde grupos minoritarios usan el idioma de la mayoría para expresar (no para perder) su identidad (Deleuze - Guattari, 1999: 28-44). Faltan análisis del fenómeno de Amereida desde la perspectiva de los estudios culturales; análisis que la sitúen en el campo de la reflexión contemporánea sobre la identidad latinoamericana. Necesitamos categorías que describan modelos complejos de la subjetividad, aquellos que no están basados en oposiciones, sino que absorben dependencias. Sirva de ejemplo la sutura, la cual, en la Antigüedad, fue un término médico y se refería al punto de sutura, al hilo que se usaba para suturar y a la propia acción de coser (del latín: suo). En su reinterpretación contemporánea, por ejemplo en el círculo de Jacques Lacan, pasó a ser un concepto ontológico ${ }^{2}$. Un ejemplo elocuente de la práctica de la sutura, de la costura del "yo poscolonial", es el famoso libro de Frantz Fanon, Piel negra, máscaras blancas. No se trata de sumar simplemente o de aunar diferentes elementos culturales, sino que se trata de unos procesos complejos que ocurren entre el sujeto y el discurso, y en los cuales son relevantes las categorías de la verdad, del otro, del subconsciente y de la identidad. Dichos procesos son variables histórica y culturalmente, oscilatorios, discontinuos y lejanos a una determinación firme y perdurable. La sutura

pone de manifiesto aquello que fue escondido (reprimido) en una lucha cuya apuesta era la (aparente) unidad. El lugar de la sutura está allí donde queda una cicatriz oculta: un elemento sensible, doloroso

\footnotetext{
${ }^{2}$ La sutura apareció en el discurso humanístico y se convirtió en un concepto ontológico a través de la conferencia de Jacques-Alain Miller La Suture (Éléments de la logique du signifiant), de 1965. En el pensamiento del maestro de Miller, Jacques Lacan, "sutura" correspondía a la idea de la unión.
} 
y deformado; es el punto en el que la identidad cosida resulta ser más fácil de romper de nuevo (Sendyka, 2015: 190).

Es importante describir y revelar las estrategias de "coser dentro de la existencia", los diferentes cortes y puntos de costura en nuestras identidades. En relación con ello, en el análisis de Amereida también parece prometedora la concepción de la exotopía de Mijaíl Bajtín, la cual, en referencia a la subjetividad, describe un estado en el que los límites de nuestras identidades discurren de una forma inesperada. Rimbaud expresó concisamente dicho fenómeno en la frase "yo es otro" (carta a Georges Izambard del 3 de mayo de 1871). De ahí que la idea más importante de la filosofía de Amereida, la de la concordancia absoluta, la consonancia entre el trabajo y la vida, "La Congruencia con La Poesía" (Iommi, 1999) 3 , no pueda obviar el hecho de que nuestro yo contenga numerosos elementos heterogéneos, diferentes y a veces espectrales. En este punto aludo a Derrida, quien, en su libro Espectros de Marx. El Estado de la deuda, el trabajo del duelo y la nueva Internacional, afirmó que, para "aprender a vivir por fin” (Derrida, 2012: 11), hay que aprender a vivir con los espectros. "Ser-con los espectros" (Derrida, 2012: 13) es una política necesaria de la memoria y de la herencia. No debemos olvidar que los encuentros y la colaboración de Latinoamérica con el Mundo se desarrollan sobre todo en el interior (la vida) del individuo.

Traducción: Justyna Cecylia Nowicka

\section{Referencias bibliográficas}

Deleuze, Gilles - Guattari, Félix (1999). Kafka. Por una literatura menor. Trans. Jorge Aguilar Mora. México: Ediciones Era.

Derrida, Jacques (2012). Espectros de Marx. El Estado de la deuda, el trabajo del duelo y la nueva Internacional. Trans. José Miguel Alarcón; Cristina de Peretti. Madrid: Trotta.

Iommi Marini, Godofredo (1999). Godo (entrevista), David Vial (transcripción). Asequible en: https://www.josevial.cl/documex/godo, fecha de consulta: 06-06-2019.

Iommi Marini, Godofredo (1982). Eneida-Amereida. Valparaíso: Escuela de Arquitectura UCV. Asequible en: http://www.memoriachilena.cl/archivos2/pdfs/MC0052571.pdf, fecha de consulta: 06-06-2019.

Mikulski, Tadeusz (1961). Temat Wrocław. Szkice ślaskie. Wrocław: Ossolineum.

\footnotetext{
${ }^{3}$ En este punto convergen dos ideas importantes de Rimbaud: el sujeto partido por dentro y absolutamente acorde en su vida con su creación, lo cual dio el resultado paradójico del abandono de la poesía por Rimbaud.
} 
Amereida: realidad cultural abierta para encuentros y colaboración

Nawrocka, Ewa (2010). Opowieści o raju utraconym. Præemiany topiki Raju w hispanoamerykańskiej powieści o selwie. Kraków: Wydawnictwo Uniwersytetu Jagiellońskiego.

Sendyka, Roma (2015). Od kultury ,ja” do kultury , siebie”. O zwrotnych formach w projektach to:̇samościonych. Kraków: Universitas. 